\title{
Development and Validation of a Novel Prognostic Model for Endometrial Cancer Based on Clinical Characteristics
}

\author{
Zhicheng Yu* \\ Sitian Wei* \\ Jun Zhang \\ Rui Shi \\ Lanfen An \\ Dilu Feng \\ Hongbo Wang (DD
}

Department of Obstetrics and Gynecology, Union Hospital, Tongji Medical College, Huazhong University of Science and Technology, Wuhan, Hubei, People's Republic of China

*These authors contributed equally to this work
Correspondence: Hongbo Wang Department of Obstetrics and Gynecology, Union Hospital, Tongji Medical College, Huazhong University of Science and Technology, Wuhan, Hubei, People's Republic of China

Tel +86 I37-2022-8749

Fax $+86027857-26114$

Email hb_wang1969@sina.com
Objective: Existing prognostic models for endometrial cancer are short of facility and effective validation. In this study, we aim to develop and validate a novel prognostic model for endometrial cancer based on clinical characteristics.

Methods: The clinical data such as age, BMI (body mass index), FIGO stage, surgical approach, myometrial invasion, grade, lymph node metastasis, pathology and menopause status were collected for constructing and validating the prognostic model from The Cancer Genome Atlas (TCGA) and Union Hospital, Tongji Medical College, Huazhong University of Science and Technology, respectively. COX regression and the least absolute shrinkage and selection operator (LASSO) COX were applied to identify the significant predictors of overall survival (OS) and construct the prognostic model. The discrimination, calibration, and clinical usefulness of the model were evaluated in both cohorts.

Results: Three hundred and sixty-seven and 286 EC patients were collected for training and validation cohort, respectively. A clinical prognostic model integrating six clinical variables including age, BMI, FIGO stage, surgical approach, myometrial invasion and grade was established. K-M analysis shows a significant difference between the low- and high-risk groups. The area under the receiver operating characteristic curve (AUC-ROC) was 0.775 (95\% CI, 0.708 to 0.843 ) and 0.870 (95\% CI, 0.758 to 0.982 ) for the training and validation cohorts which indicating reliable discrimination. The calibration curve revealed excellent predictive accuracy and the Hosmer-Lemeshow test also verified this. Decision curve analysis (DCA) for the prognostic model indicated that it would add more benefits than either the detect-all-patients scheme or the detect-none scheme. In addition, our model has a superior AUC comparing with any single factor as predicting OS.

Conclusion: Our predictive model offers a convenient and accurate tool for clinicians to estimate the prognosis of EC patients.

Keywords: clinical characteristics, endometrial cancer, prognostic model, TCGA

\section{Introduction}

Endometrial carcinoma (EC), as the malignant epithelial tumors of the endometrium, is one of the three most common malignant tumors in the female reproductive system. ${ }^{1}$ The incidence rate of EC is increasing ${ }^{2,3}$ and shows a younger trend in the past 20 years while $70-75 \%$ of the patients are postmenopausal women, with an average age of 55 years. ${ }^{4}$ At present, surgery is the first-line treatment for the disease, while radiotherapy and chemotherapy are only used as adjuvant treatment. ${ }^{5}$ The 5-years overall survival of EC decreased dramatically when metastasis or 
relapse. ${ }^{6}$ Therefore, it is imperative to find effective prognostic characteristics of EC to instruct appropriate management.

As we all know, the prognosis of EC is influenced by age, ${ }^{7}$ BMI,${ }^{8,9}$ FIGO stage, ${ }^{10}$ and other clinical factors, ${ }^{11}$ but the exact degree of correlation is underdetermined. It is also inappropriate to use a single factor to predict overall survival due to the heterogeneity of EC patients. ${ }^{12,13}$ Some studies have constructed prognostic models for EC based on clinical and transcriptome variables, ${ }^{14}$ but lack effective external validation. We can conclude that the predictive models based on transcriptomes have relatively better discrimination and calibration rather based on clinical variables. However, it is inconvenient to apply the transcriptome models to a large-scale population. For this reason, through combining simple clinical indicators such as age at diagnosis, BMI, grade, FIGO stage, surgical approach, and myometrial invasion, we established a personalized prognosis model for EC patients to obtain predictive information.

In this study, we focused on the clinical variables alterations of The Cancer Genome Atlas (TCGA) EC patients to set up a complete prognostic model to predict prognosis. In addition, we used multiple sets of clinical data to verify the model and to prove its effectiveness, which can provide a theoretical basis for the prognosis risk assessment of EC patients.

\section{Methods}

\section{Data Collection and Filtering}

We reviewed two independent cohorts diagnosed with EC which the primary cohort contains clinicopathologic and survival information were derived from TCGA database (https://gdc-portal.nci.nih.gov/) in Dec 2020 and the validation cohort was retrospectively collected from Union Hospital, Tongji Medical College, Huazhong University of Science and Technology between Feb 2012 and Dec 2020. Patients were excluded from this study for these reasons, i. the history of chemoradiotherapy before surgery, ii. without follow-up information, iii. incomplete clinical data. The variables selected to be initially analyzed in the study were: age, BMI (body mass index), FIGO stage, surgical approach, myometrial invasion, grade, lymph node metastasis, pathology and menopause status. This study was approved by ethics committee of Tongji Medical College, Huazhong University of Science and Technology (No. 2021-S046).

\section{Development of the Prognostic Model}

The 9 clinical indicators were firstly analyzed with univariate Cox regression (the 2-sided Log rank test). Multivariate Cox regression analysis ( $\alpha$ in $=0.05$, aout $=$ 0.10) was applied for variables with a univariate Cox regression $\mathrm{P}<0.05$. Those indicators were considered to be risk factors when the hazard ratio (HR) greater than 1 and protective factors when less than 1 .

Lasso Cox analysis found the clinical indicators that were most correlated with overall survival to prevent overfitting and 10 rounds of cross-validation were performed. The Lasso regression was run for 1000 times and randomly stimulated for 1000 times for each cycle. The risk score was then calculated for each patient based on the clinical indicators. The formula is as follows:

$$
\text { Risk score }=\sum_{i=1}^{n} \text { coef clinical indicators }
$$

The median value of risk score was considered as the cut-off point to distinguish the high-risk group from the low-risk group. To provide clinicians a convenient quantitative prognostic model, we constructed the nomogram on the basis of filtered variables. In addition, Kaplan-Meier survival analysis was used to compare the survival differences between different risk groups in the two cohorts.

\section{Validation of the Prognostic Model}

For the discrimination validation, we calculated the areas under the time-dependent ROC curves (AUC-ROC) of the prognostic models. Regarding to calibration, the calibration curve was constructed. We performed HosmerLemeshow test to evaluate the calibration of the prognostic model. Finally, decision curve analysis (DCA) was used to explore the clinical net benefit of the nomogram.

\section{Statistical Analysis}

Statistical analysis was processed by $\mathrm{R}$ version 4.0.5 (Institute for Statistics and Mathematics, Vienna, Austria; https://www.r-project.org). (Packages: survival, glmnet, rms, survival ROC). The continuous variables were transformed into binary variables as shown in Table 1 and Table S1 and subsequently described in terms of counts and percentages. $\mathrm{P}<0.05$ was considered statistically significant.

\section{Results}

\section{The Clinical Characteristics of Patients}

We eventually obtained 367 and 286 EC patients for the training and validation cohorts respectively after quality 
Table I The Clinical Characteristics of EC Patients in the Training Cohort

\begin{tabular}{|c|c|c|c|c|c|}
\hline \multirow[t]{2}{*}{ Variables } & \multirow[t]{2}{*}{$\mathbf{N}(\%)$} & \multicolumn{2}{|c|}{ Univariate Cox } & \multicolumn{2}{|c|}{ Multivariate Cox } \\
\hline & & HR (95\% Cl) & P-value & HR (95\% Cl) & P-value \\
\hline \multicolumn{6}{|l|}{ Age } \\
\hline$<60$ & $121(33.0)$ & \multicolumn{2}{|l|}{ Reference } & & \\
\hline$\geq 60$ & $246(67.0)$ & I.584(0.866-2.898) & 0.136 & & \\
\hline \multicolumn{6}{|l|}{ BMI } \\
\hline$<30$ & $146(40.0)$ & \multicolumn{2}{|l|}{ Reference } & & \\
\hline$\geq 30$ & $221(60.0)$ & $\mathrm{I} .42 \mathrm{I}(0.826-2.443)$ & 0.204 & & \\
\hline \multicolumn{6}{|l|}{ FIGO } \\
\hline I & $235(64.0)$ & \multicolumn{2}{|l|}{ Reference } & \multicolumn{2}{|l|}{ Reference } \\
\hline II & $31(8.4)$ & $0.339(0.046-2.525)$ & 0.291 & $0.233(0.03|-| .75 \mid)$ & 0.157 \\
\hline III & $82(22.4)$ & $5.041(2.956-9.866)$ & $<0.001$ & $4.114(2.163-7.823)$ & $<0.001$ \\
\hline IV & $19(5.2)$ & $8.238(3.807-17.830)$ & $<0.001$ & $9.012(1.712-47.442)$ & 0.009 \\
\hline \multicolumn{6}{|l|}{ Surgical approach } \\
\hline Minimally invasive & 154(42) & \multicolumn{2}{|l|}{ Reference } & & \\
\hline Open & $213(58)$ & $0.837(0.492-1.424)$ & 0.512 & & \\
\hline \multicolumn{6}{|l|}{ Myometrial invasion } \\
\hline$<50 \%$ & $200(54.5)$ & \multicolumn{2}{|l|}{ Reference } & \multicolumn{2}{|l|}{ Reference } \\
\hline$\geq 50 \%$ & $167(45.5)$ & $2.161(1.262-3.699)$ & 0.005 & I.44I (0.809-2.567) & 0.215 \\
\hline \multicolumn{6}{|l|}{ Grade } \\
\hline I & $73(19.9)$ & \multicolumn{2}{|l|}{ Reference } & \multicolumn{2}{|l|}{ Reference } \\
\hline 2 & $88(24.0)$ & $10.739(1.385-83.274)$ & 0.023 & $9.789(1.256-76.308)$ & 0.029 \\
\hline 3 & $206(56.1)$ & $19.058(2.627-138.244)$ & 0.004 & II.58I(I.564-85.756) & 0.016 \\
\hline \multicolumn{6}{|c|}{ Lymph node metastasis } \\
\hline Yes & $68(18.5)$ & \multicolumn{2}{|l|}{ Reference } & & \\
\hline No & $299(81.5)$ & $1.607(0.887-2.910)$ & 0.118 & & \\
\hline \multicolumn{6}{|l|}{ Pathology } \\
\hline EAC & $277(75.5)$ & \multicolumn{2}{|l|}{ Reference } & & \\
\hline NEAC & $90(24.5)$ & $1.04 I(0.570-1.901)$ & 0.895 & & \\
\hline \multicolumn{6}{|l|}{ Menopause status } \\
\hline Pre & $28(7.6)$ & \multicolumn{2}{|l|}{ Reference } & \multicolumn{2}{|l|}{ Reference } \\
\hline Post & $339(92.4)$ & $0.253(0.133-0.481)$ & $<0.001$ & $1.560(0.366-6.642)$ & 0.548 \\
\hline
\end{tabular}

Abbreviations: EC, endometrial cancer; BMI, body mass index; FIGO, international federation of gynecology and obstetrics; EAC, endometrial adenocarcinoma; NEAC, non-endometrial adenocarcinoma. 
control as mentioned above. The clinical characteristics of enrolled EC patients in training and validation cohort are shown in Table 1 and Table S1. Univariate Cox proportional hazards regression and subsequent multivariate regression were used to calculate the clinical variables in the training and validation cohort. FIGO stage, myometrial invasion, grade and menopause status were identified as risk factors $(\mathrm{HR}>1)$ for prognosis in both cohorts while multivariate analysis indicating distinct indicators.

\section{Identifying Variables and Construction of Prognostic Model}

To prevent over-fitting, lasso Cox analysis was used to identify variables that were most correlated with overall survival (Figure 1A) and 10 rounds of cross-validation were further performed to determine the optimal value of the penalty parameter (Figure 1B). A cox proportional hazards model was established based on 6 variables after lasso regression analysis. The risk score in our prognostic model was a sum of each feature after weighted and the formula was as follows: risk score $=0.275$ (if age $\geq 60$ years old $)+(0.623 *$ FIGO stage) +0.283 (if $\mathrm{BMI} \geq 30 \mathrm{~kg} / \mathrm{m}^{2}$ ) -0.216 (if the surgical approach was open) +0.235 (if myometrial invasion $\geq 50 \%$ ) $+(0.525 *$ grade $)$. In addition, we enrolled the above variables and presented as the nomogram to visualize the prognostic model (Figure 2). The K-M analysis was also show that the survival time were extremely discrepant between two risk groups in the training and validation cohorts (Figure S1A and $\underline{B}$ ). This indicates the excellent predictive power of the prognostic model.

\section{Validation of the Prognostic Model}

To validate the prognostic model, we calculated the discrimination and calibration of the prognostic models in both cohorts. For internal validation, the ROC curve yielded an AUC of 0.775 (95\% CI, 0.708 to 0.843 ) (Figure 3A). The calibration curve of the prognostic model for the probability of 1, 3, 5-yr survival showed no deviations between prediction and observation in the training cohort (Figure $4 \mathrm{~A}-\mathrm{C}$ ) and the Hosmer-Lemeshow test showed that there were no departure from perfect fit $(\mathrm{P}=0.725)$. For independent validation, the AUC was 0.870 (95\% CI, 0.758 to 0.982 ) (Figure 3B) which indicating a great discriminative ability. The calibration curve also revealed excellent predictive accuracy (Figure 4D-F) and the Hosmer-Lemeshow test revealed there was non-significant statistic $(\mathrm{P}=0.793)$.

\section{Clinical Application}

Decision curve analysis (DCA) for the prognostic model was performed in the training and validation cohorts (Figure 5A and B) to identify the clinical utility via calculating the net benefits at different threshold probabilities. The net benefit was quantified by subtracting the proportion of patients who are false positive from true positive. The black and blue lines represent two extreme conditions, the former indicating that all samples are negative and the net benefit is 0 , the latter indicating that all samples are positive, and the net benefit is a negative anticline. We can conclude that the prognostic model would add more benefit than either the detect-all-patients or the detect-none scheme. ROC curves of $1,3,5$-yr were also conducted in both cohorts to verified the
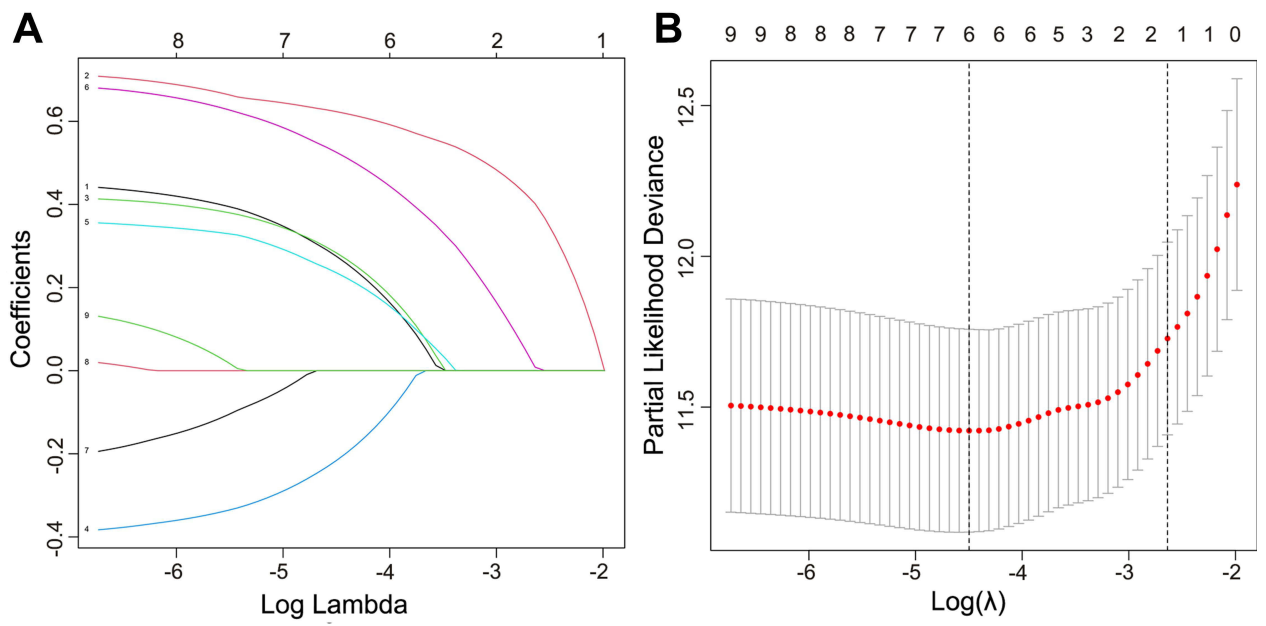

Figure I Identifying the prognostic variables of the overall survival (OS) using the Least absolute shrinkage and selection operator (LASSO) COX. (A) LASSO coefficients of the whole factors included into analysis. (B) Tuning parameter identification using the minimum criteria. The dotted vertical line was drawn at the optimal value choose by the 10 -fold cross-validation based on the minimum criteria (the smallest partial likelihood deviance). 


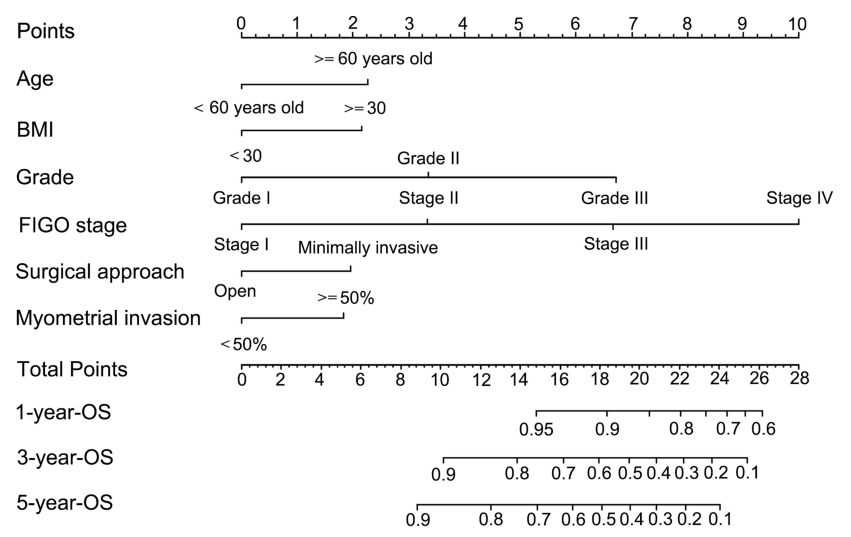

Figure 2 Nomogram that predicts the overall survival (OS) of EC patients.

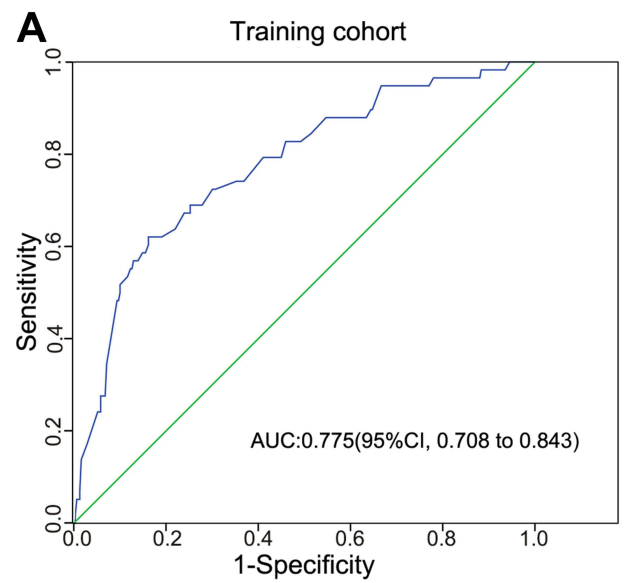

specificity and sensitivity of our model (Figure S2A and B). In addition, the prognostic model has the maximal AUC comparing with individual variable in both cohorts which indicating a better predictive ability (Figure S2C and $\underline{\text { D) }}$.

\section{Discussion}

It is of great importance to obtain the prognostic information before starting treatment. At present, researchers have been searching for EC prognosis-related clinical variables and establishing EC prognostic prediction model with higher accuracy to provide better clues for evaluating reliable individualized prognosis, thereby improving the

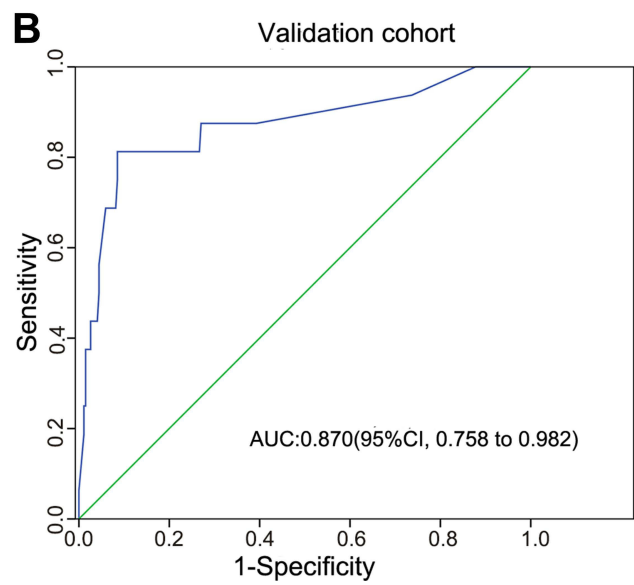

Figure 3 Receiver operating characteristic (ROC) curve of the prognosis model in each cohort. (A) represent ROC curve of our prognosis model in the training cohort; (B) represent the ROC curve of model in the validation cohort.
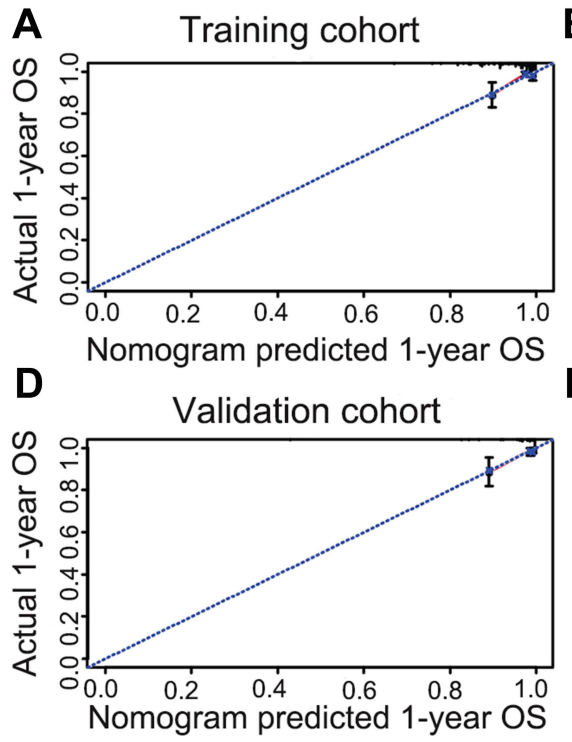

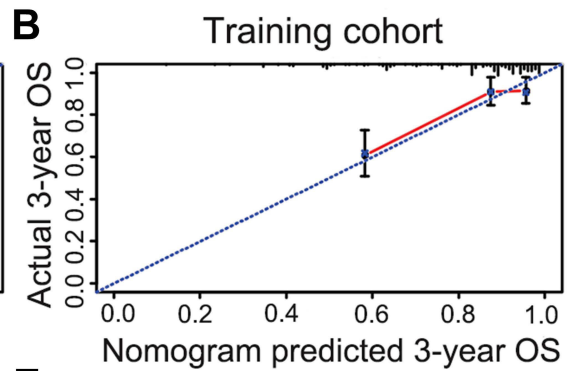

E

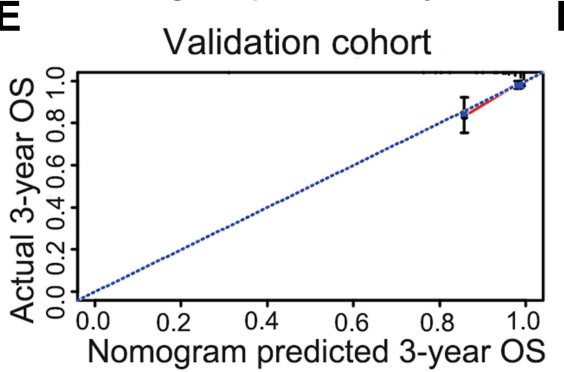

C Training cohort

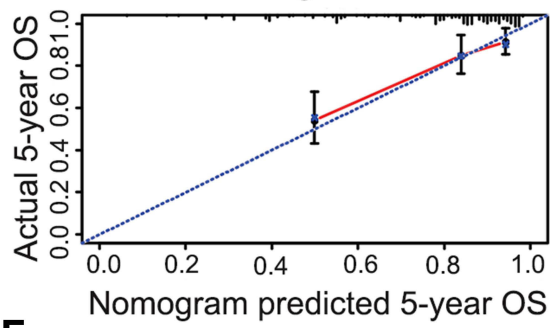

\section{F}

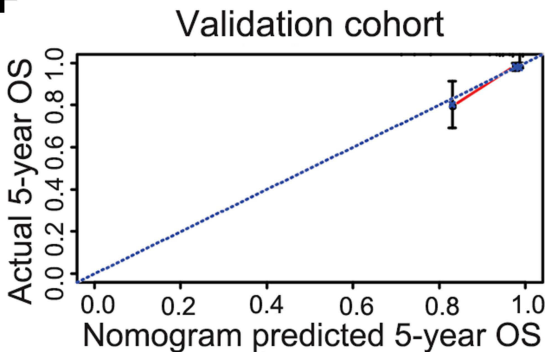

Figure 4 Calibration curves of OS at different time points (I-, 3- and 5-yr) in each cohort. (A-C) represent calibration curves of OS in the training cohort; (D-F) represent calibration curves of OS in the validation cohort. 

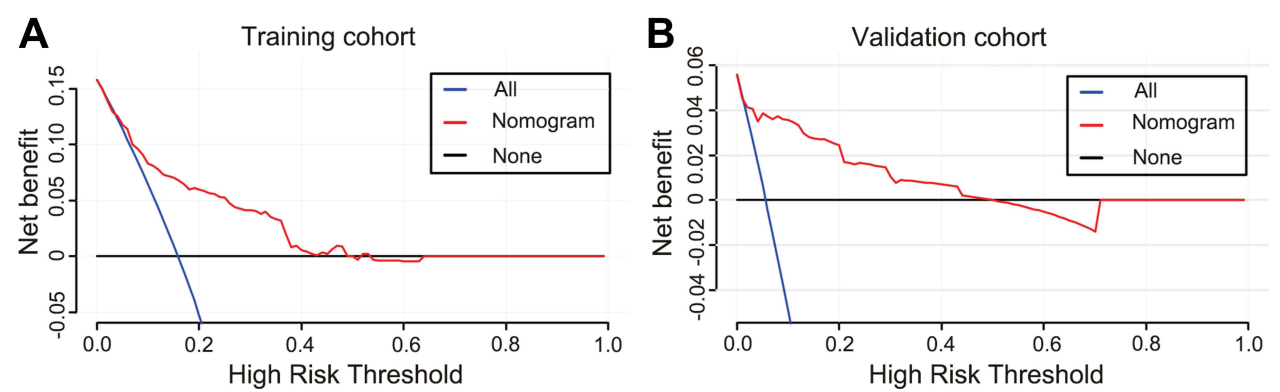

Figure 5 Decision curve analyses of the model predicting overall survival (OS) at I-, 3-and 5-yr. X-axis shows different thresholds. Y-axis represents the net benefit. Net benefit was counted as summing the true positives and subtracting the false positives. The black horizontal line assumes that no patients died whereas the blue line assumes all cases dead. (A and $\mathbf{B}$ ) represent the decision curve of our nomograms in the training and validation cohort.

overall survival of EC patients. ${ }^{15,16}$ Previous studies have shown that the clinical variables related to the prognosis of EC include pathological grade, pathological stage, FIGO stage, age at diagnosis, degree of muscular invasion, vascular tumor thrombus, and lymph node metastasis. ${ }^{17-20}$ Our study used lasso Cox analysis and identified 6 factors that were most related to EC prognosis, including the age at diagnosis, BMI, grade, FIGO stage, surgical approach, and myometrial invasion, after acquiring the clinical data of EC patients from the TCGA database. Based on the above 6 variables, a cox proportional hazards model was established. The risk score in our prognostic model was a sum of each feature after weighted and the formula was as follows: risk score $=0.275$ (if age $\geq 60$ years old) + $(0.623 *$ FIGO stage $)+0.283$ (if BMI $\geq 30 \mathrm{~kg} / \mathrm{m} 2)-0.216$ (if the surgical approach was open) +0.235 (if myometrial invasion $\geq 50 \%)+(0.525 *$ grade $)$. After that, we enrolled the above variables and presented them as the nomogram to visualize the prognostic model. The K-M analysis also showed that the survival time was extremely discrepant between two risk groups in the training and validation cohorts, which indicated the excellent predictive power of the prognostic model.

It is well acknowledged that the age of initial diagnosis has been proved to be associated with EC prognosis. ${ }^{21}$ Furthermore, recent studies have indicated that $\mathrm{BMI}^{22}$ and pathological grade are also effective predictors for EC. $^{23}$ A retrospective study elucidated that morbidly obese women had higher mortality rates compared with women with a normal BMI while a systematic review found that the progression-free survival and diseasespecific mortality were not associated with obesity. ${ }^{24,25}$ Also, some evidence has indicated that the FIGO stage not only acts as a guideline for treatment, but also has great potentials as an indicator for prognosis. ${ }^{26}$ In the past decades, surgical approaches were under controversy all the time. Ramirez et al and Melamed et al have elucidated that the benefits for cervical cancer patients who underwent open surgery were more than minimally invasive surgery. ${ }^{27,28}$ With regard to EC patients, the effects on the prognosis of surgical approaches are also underdetermined. A comprehensive meta-analysis including 4389 EC patients has indicated that the OS and DFS show no significant difference between laparoscopy and laparotomy. $^{29}$ However, minimally invasive surgical approach has its strength for reducing blood loss, length of hospital stay, and the incidence and severity of surgical complications. $^{30}$ Long-term follow-up and large-scale cases are necessary to determine which surgical approach has a better prognosis. Additionally, myometrial invasion is also an independent variable for the prognostic outcome of EC. ${ }^{31,32}$

Numbers of studies have established different prognostic models based on clinical and transcriptome characteristics for EC patients. Deng et al identified 28 EC prognosis-related RNAs and constructed a reliable prognostic model. ${ }^{14}$ And Fan et al thoroughly investigated the implications of metabolism-related genes in endometrial cancer progression. ${ }^{33}$ While those models based on transcriptome have reliable discrimination and calibration, it is not universal to apply them in clinical practice. Based on this, the prognostic models on the strength of the clinical variables perform superior convenience and EC patients do not need redundant examination such as molecular diagnosis or genomic sequence. Moreover, we provided a simple nomogram to visualize the model so that clinicians could employ it handily. Its simplicity will allow clinicians to quickly evaluate survival outcomes and make optimal decisions about individual EC patients. Even individuals without 
a medical background can easily read the meaning of our nomogram. Those features will make our model an accurate and feasible tool for clinicians and EC patients to get the prognosis information in advance.

There are also some limitations in our study. First of all, it owns the weakness of retrospective studies. Second, TCGA data lacks more comprehensive clinical information such as specific treatment schedules which may have an impact on the prognosis. Next, our model does not include patient race which in some studies has been suggested as an important prognostic factor. ${ }^{34}$ In addition, the single-center derived validation cohort is also insufficient to verify our prognostic model. Overall, our study performed a convenient and reliable tool for clinicians to estimate prognosis and choose an optimal therapeutic schedule.

\section{Conclusion}

In conclusion, our predictive model integrating several routine clinical variables offers a convenient and accurate tool for clinicians to estimate the prognosis of EC patients. Additionally, we used multiple sets of clinical data to verify the model and to prove its effectiveness which can provide a theoretical basis for the prognosis risk assessment of EC patients.

\section{Ethics Approval and Consent to Participate}

This study was approved by ethics committee of Tongji Medical College, Huazhong University of Science and Technology (No. 2021-S046). The patients have signed their informed consent to participate in this study.

\section{Acknowledgments}

This work was supported by Wuhan Science and Technology Bureau of Hubei Province of China (2019020701011430) and Major Technical Innovation Project in Hubei Province of China (2019ACA138).

\section{Disclosure}

The authors report no conflicts of interest in this work.

\section{References}

1. Sung H, Ferlay J, Siegel RL, et al. Global cancer statistics 2020: GLOBOCAN estimates of incidence and mortality worldwide for 36 cancers in 185 countries. CA Cancer J Clin. 2021;71 (3):209-249.
2. Bray F, Ferlay J, Soerjomataram I, Siegel RL, Torre LA, Jemal A. Global cancer statistics 2018: GLOBOCAN estimates of incidence and mortality worldwide for 36 cancers in 185 countries. CA Cancer J Clin. 2018;68:394-424. doi:10.3322/caac.21492

3. Torre LA, Islami F, Siegel RL, Ward EM, Jemal A. Global cancer in women: burden and trends. Cancer Epidemiol Biomarkers Prev. 2017;26:444-457. doi:10.1158/1055-9965.EPI-16-0858

4. Lortet-Tieulent J, Ferlay J, Bray F, Jemal A. International patterns and trends in endometrial cancer incidence, 1978-2013. J Natl Cancer Inst. 2018;110:354-361. doi:10.1093/jnci/djx214

5. Koh WJ, Abu-Rustum NR, Bean S, et al. Uterine neoplasms, version 1.2018, NCCN clinical practice guidelines in oncology. $J$ Natl Compr Canc Netw. 2018;16:170-199. doi:10.6004/ jncen.2018.0006

6. Colombo N, Creutzberg C, Amant F, et al. ESMO-ESGO-ESTRO consensus conference on endometrial cancer: diagnosis, treatment and follow-up. Ann Oncol. 2016;27:16-41. doi:10.1093/annonc/ mdv484

7. Okuma K, Yamashita H, Kawana K, Nakagawa S, Oda K, Nakagawa K. Advanced age is a significant determinant of poor prognosis in patients treated with surgery plus postoperative radiotherapy for endometrial cancer. $J$ Obstet Gynaecol Res. 2010;36:757-763. doi:10.1111/j.1447-0756.2010.01202.x

8. Raglan O, Kalliala I, Markozannes G, et al. Risk factors for endometrial cancer: an umbrella review of the literature. Int $J$ Cancer. 2019;145:1719-1730. doi:10.1002/ijc.31961

9. Modesitt SC, van Nagell JR. The impact of obesity on the incidence and treatment of gynecologic cancers: a review. Obstet Gynecol Surv. 2005;60:683-692. doi:10.1097/01.ogx.0000180866.62409.01

10. Scharl S, Gerken M, Sprötge T, et al. Comparison of survival outcomes and effects of therapy between subtypes of high-grade endometrial cancer - a population-based study. Acta Oncologica. 2021;60:1-7.

11. Han KH, Kim HS, Lee M, Chung HH, Song YS. Prognostic factors for tumor recurrence in endometrioid endometrial cancer stages IA and IB. Medicine (Baltimore). 2017;96:e6976. doi:10.1097/ MD.000000000006976

12. Frederick PJ, Straughn JM. The role of comprehensive surgical staging in patients with endometrial cancer. Cancer Control. 2009;16:23-29. doi:10.1177/107327480901600104

13. Amant F, Moerman P, Neven P, Timmerman D, Van Limbergen E, Vergote I. Endometrial cancer. Lancet. 2005;366:491-505. doi:10.1016/S0140-6736(05)67063-8

14. Deng F, Mu J, Qu C, et al. A novel prognostic model of endometrial carcinoma based on clinical variables and oncogenomic gene signature. Front Mol Biosci. 2020;7:587822. doi:10.3389/ fmolb.2020.587822

15. Prat J. Prognostic parameters of endometrial carcinoma. Hum Pathol. 2004;35:649-662. doi:10.1016/j.humpath.2004.02.007

16. Kolehmainen A, Pasanen A, Tuomi T, Koivisto-Korander R, Bützow R, Loukovaara M. Clinical factors as prognostic variables among molecular subgroups of endometrial cancer. PLoS One. 2020;15:e0242733. doi:10.1371/journal.pone.0242733

17. Braun MM, Overbeek-Wager EA, Grumbo RJ. Diagnosis and management of endometrial cancer. Am Fam Physician. 2016;93:468-474.

18. Singh N, Hirschowitz L, Zaino R, et al. Pathologic prognostic factors in endometrial carcinoma (other than tumor type and grade). Int J Gynecol Pathol. 2019;38(Suppl 1):S93-S113. doi:10.1097/ PGP.0000000000000524

19. Uharcek P. Prognostic factors in endometrial carcinoma. J Obstet Gynaecol Res. 2008;34:776-783. doi:10.1111/j.14470756.2008.00796.x

20. Kandoth C, Schultz N, Cherniack AD, et al. Integrated genomic characterization of endometrial carcinoma. Nature. 2013;497:67-73. doi:10.1038/nature12113 
21. Alektiar KM, Venkatraman E, Abu-Rustum N, Barakat RR. Is endometrial carcinoma intrinsically more aggressive in elderly patients? Cancer. 2003;98:2368-2377. doi:10.1002/cncr.11830

22. Secord AA, Hasselblad V, Von Gruenigen VE, et al. Body mass index and mortality in endometrial cancer: a systematic review and meta-analysis. Gynecol Oncol. 2016;140:184-190. doi:10.1016/j. ygyno.2015.10.020

23. Abu-Rustum NR, Zhou Q, Gomez JD, et al. A nomogram for predicting overall survival of women with endometrial cancer following primary therapy: toward improving individualized cancer care. Gynecol Oncol. 2010;116:399-403. doi:10.1016/j.ygyno.2009.11.027

24. von Gruenigen VE, Tian C, Frasure H, Waggoner S, Keys H, Barakat RR. Treatment effects, disease recurrence, and survival in obese women with early endometrial carcinoma: a Gynecologic Oncology Group study. Cancer. 2006;107(12):2786-2791. doi:10.1002/cncr.22351

25. Arem H, Irwin ML. Obesity and endometrial cancer survival: a systematic review. Int $J$ Obes (Lond). 2013;37(5):634-639. doi:10.1038/ijo.2012.94

26. Creasman WT, Odicino F, Maisonneuve P, et al. Carcinoma of the corpus uteri. FIGO 26th annual report on the results of treatment in gynecological cancer. Int J Gynaecol Obstet. 2006;95(Suppl 1): S105-S143. doi:10.1016/S0020-7292(06)60031-3

27. Ramirez PT, Frumovitz M, Pareja R, et al. Minimally invasive versus abdominal radical hysterectomy for cervical cancer. $N$ Engl J Med. 2018;379:1895-1904. doi:10.1056/NEJMoa1806395
28. Melamed A, Margul DJ, Chen L, et al. Survival after minimally invasive radical hysterectomy for early-stage cervical cancer. $N$ Engl J Med. 2018;379:1905-1914. doi:10.1056/NEJMoa1804923

29. Galaal K, Donkers H, Bryant A, Lopes AD. Laparoscopy versus laparotomy for the management of early stage endometrial cancer. Cochrane Database Syst Rev. 2018;10(10):CD006655. doi:10.1002/ 14651858.CD006655.pub3

30. Frumovitz M, Escobar P, Ramirez PT. Minimally invasive surgical approaches for patients with endometrial cancer. Clin Obstet Gynecol. 2011;54(2):226-234. doi:10.1097/GRF.0b013e318218637d

31. Orezzoli JP, Sioletic S, Olawaiye A, Oliva E, Del Carmen MG. Stage II endometrioid adenocarcinoma of the endometrium: clinical implications of cervical stromal invasion. Gynecol Oncol. 2009;113:316-323. doi:10.1016/j.ygyno.2009.03.007

32. Homesley HD. Revised 1988 International Federation of Gynecology and Obstetrics staging systems for endometrial and vulvar cancer: an assessment. Clin Obstet Gynecol. 1992;35:89-94. doi:10.1097/ 00003081-199203000-00012

33. Fan Y, Li X, Tian L, Wang J. Identification of a metabolism-related signature for the prediction of survival in endometrial cancer patients. Front Oncol. 2021;11:630905. doi:10.3389/fonc.2021.630905

34. Wright JD, Fiorelli J, Schiff PB, et al. Racial disparities for uterine corpus tumors: changes in clinical characteristics and treatment over time. Cancer. 2009;115:1276-1285. doi:10.1002/cncr.24160

\section{Publish your work in this journal}

Cancer Management and Research is an international, peer-reviewed open access journal focusing on cancer research and the optimal use of preventative and integrated treatment interventions to achieve improved outcomes, enhanced survival and quality of life for the cancer patient.
The manuscript management system is completely online and includes a very quick and fair peer-review system, which is all easy to use. Visit http://www.dovepress.com/testimonials.php to read real quotes from published authors. 